\title{
Dynamic Pull-In Investigation of a Clamped-Clamped Nanoelectromechanical Beam under Ramp-Input Voltage and the Casimir Force
}

\author{
Amir R. Askari and Masoud Tahani \\ Department of Mechanical Engineering, Ferdowsi University of Mashhad, Mashhad 91775-1111, Iran \\ Correspondence should be addressed to Amir R. Askari; amaskari@gmail.com
}

Received 19 October 2012; Accepted 19 November 2012; Published 14 May 2014

Academic Editor: Hamid Mehdigholi

Copyright (C) 2014 A. R. Askari and M. Tahani. This is an open access article distributed under the Creative Commons Attribution License, which permits unrestricted use, distribution, and reproduction in any medium, provided the original work is properly cited.

The influence of the Casimir excitation on dynamic pull-in instability of a nanoelectromechanical beam under ramp-input voltage is studied. The ramp-input actuation has applications in frequency sweeping of RF-N/MEMS. The presented model is nonlinear due to the inherent nonlinearity of electrostatics and the Casimir excitations as well as the geometric nonlinearity of midplane stretching. A Galerkin based reduced order modeling is utilized. It is found that the calculated dynamic pull-in ramp input voltage leads to dynamic pull-in step input voltage by increasing the slope of voltage-time diagram. This fact is utilized to verify the results of present study.

\section{Introduction}

Nano/microelectromechanical systems (N/MEMS) are mostly used as sensors and actuators. Because of their small size, low power consumption, and the reliability of batch fabrications, there are lots of potential applications in engineering. Clamped-clamped microbeams represent major structural components and play crucial roles in these systems. One of the most important phenomena associated with electrostatically actuated N/MEMS is pull-in instability which occurs when input voltage exceeds its critical value. In this manner, the movable part is suddenly collapsed toward the substrate. This phenomenon was observed experimentally by many researchers. Nathanson et al. [1] and Taylor [2] have investigated this phenomenon experimentally. This instability can occur in both static and dynamic circumstances. If the rate of applied voltage is negligible, the static pull-in instability may be observed; otherwise, one can observe DC dynamic pull-in.

At the nanoscale, the intermolecular forces significantly influence dynamics of nanobeams. The Casimir effect is the most important force at the scale of N/MEMS. It represents attractive force between two flat parallel plates of solids that arises from quantum fluctuations in the ground state of the electromagnetic field [3]. The Casimir interaction becomes operative at separations less than several micrometers and above $20 \mathrm{~nm}$ [4]. The influence of Casimir force on the pull-in instability of nano- and microsystems has been investigated by many researchers. Lin and Zhao [5] studied the influence of the Casimir force on static pull-in behaviour of nanoelectromechanical systems using lumped model. Ramezani et al. [6] proposed a distributed parameter model to study the static pull-in instability of nanocantilevers subjected to intermolecular and electrostatic forces. They transferred nonlinear differential equation of the model into the integral form by using Green's function of the cantilever beam. Koochi et al. [7] investigated the effect of the Casimir attraction on the nonlinear pull-in behaviour of cantilever and double cantilever NEMS using modified Adomian decomposition method (MAD). They neglected the effect of inertia and the von Kármán nonlinearity; in other words, they have investigated the static pull-in case. Dynamic pull-in instability of electrically actuated microbeams in presence of the Casimir force has been investigated by Moghimi Zand and Ahmadian [8]. They consider the von Kármán nonlinearity of midplane stretching, applied axial loading, fringing field effect, and the 


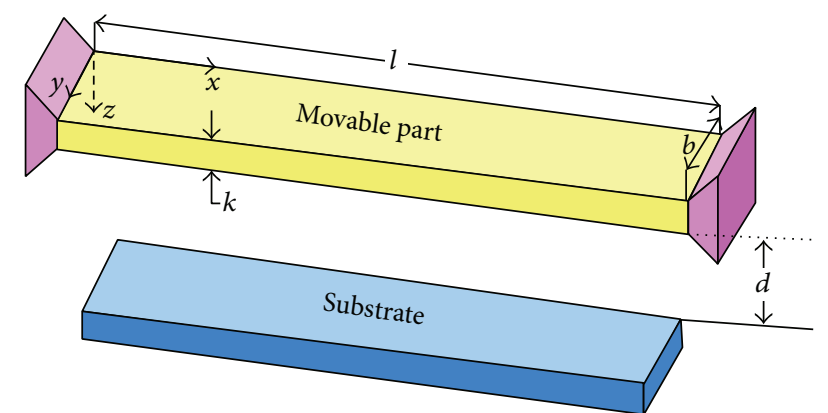

FIGURE 1: Schematic of a ramp-input voltage actuated clampedclamped nano/microbeam under the effect of Casimir force.

Casimir attraction and solved the governing equation using nonlinear finite element method (NFEM).

Most of the studies on microstructures have been performed using step-input and harmonic actuations. However, other actuation shapes also have application in microstructures. Ramp-input actuation has applications in frequency sweeping and contact time study of RF-MEMS. Contact time is defined as the time taken by a microstructure to move from the initial position to the position where the deformable part contacts the substrate. It is noted that the interaction of Casimir force and ramp voltage excitation has not been investigated to date.

In present study, the governing equation of motion of nano/microbeams under the combined effect of electrostatic excitation due to ramp-input voltage and the Casimir force has been derived. This model is nonlinear due to the inherent nonlinearity of electrostatic excitation, Casimir attraction, and the geometric nonlinearity of the von Kármán midplane stretching. A numerical analytical method based on Galerkin reduced order modelling has been used to convert the partial differential equation of motion to a set of ordinary differential equations in order to investigate the nonlinear response of double clamped nano/microbeams. The results are in good agreement with those presented in the literature for dynamic pull-in case due to the step input voltage.

\section{Modelling and Formulation}

Consider a fully clamped nano/microbeam of length $l$, width $b$, thickness $k$, and density $\rho$ under the combined action of the electrostatic excitation due to ramp-input voltage and the Casimir force (Figure 1). The distance between the beam and the stationary electrode is $d$. Also, $x, y$, and $z$ are, respectively, the coordinate along the length, width, and thickness. $W$ is deflection, $t$ is time, $I$ is the moment of inertia of the crosssection about the $y$ axis, $v$ is Poisson's ratio, and $E$ is the effective Young modulus of the nano/microbeam which is replaced by $E /\left(1-v^{2}\right)$ when $b>5 k$.

The electrostatic excitation by ramp-input DC voltage $v_{\mathrm{DC}}$ per unit length of the beam can be expressed as

$$
F_{\mathrm{es}}=\frac{\varepsilon b v_{\mathrm{DC}}^{2}}{2(d-W)^{2}}
$$

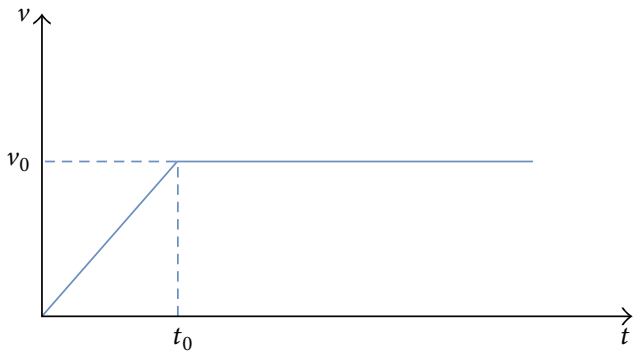

Figure 2: Ramp-input voltage.

where

$$
v_{\mathrm{DC}}=\frac{v_{0}}{t_{0}} t U\left(t_{0}-t\right)+v_{0} U\left(t-t_{0}\right),
$$

where $v_{0}$ is the maximum value of input voltage and $t_{0}$ is the duration in which this voltage is applied (see Figure 2) and $U(t)$ is the unit step function.

The Casimir force per unit length of the beam takes the following form [9]:

$$
f_{\text {cas }}=\frac{\pi^{2} \hbar c b}{240(d-W)^{4}}
$$

where $\varepsilon$ is dielectric constant of medium, $\hbar=1.055 \times 10^{-34} \mathrm{~J}$ is Planck's constant divided by $2 \pi$, and $c=2.998 \times 10^{8} \mathrm{~m} / \mathrm{s}$ is the speed of light in vacuum.

Due to the elongation of fixed-fixed nano/microbeam which is called the midplane stretching effect and the mismatch of both thermal expansion coefficient and crystal lattice period between substrate and microbeam film which is unavoidable in surface micromachining techniques, the resultant axial force is applied to the nano/microbeam [10]:

$$
F_{\text {axial }}=F_{r}+F_{a} .
$$

The axial force due to the midplane stretching effect takes the following form [11]:

$$
F_{a}=\frac{E b k}{2 l} \int_{0}^{1} W^{\prime 2} d x
$$

and the one due to the residual stress can be defined as

$$
F_{r}=\sigma_{r} b k .
$$

So the equation of motion of the clamped-clamped nano/microbeam subjected to the combined effect of ramp-input voltage and the Casimir force is as follows:

$$
\begin{aligned}
& E I W^{\prime \prime \prime \prime}+f \dot{W}+\rho b k \ddot{W} \\
& \quad=\left(F_{r}+\frac{E b k}{2 l} \int_{0}^{1} W^{\prime 2} d x\right) W^{\prime \prime}+F_{\mathrm{es}}+F_{\mathrm{cas}},
\end{aligned}
$$

where dot and prime signs denote derivatives with respect to $t$ and $x$, respectively, $f$ is equivalent viscose damping coefficient per unit length of the beam due to squeeze film 
damping [12]. The nano/microbeam deflection is subjected to the following kinematic boundary conditions:

$$
\begin{aligned}
W(0, t)=0 ; & \frac{\partial W(0, t)}{\partial x}=0 ; \\
W(l, t)=0 ; & \frac{\partial W(l, t)}{\partial x}=0 .
\end{aligned}
$$

The initial conditions are assumed as follows:

$$
W(x, 0)=0 ; \quad \frac{\partial W(x, 0)}{\partial t}=0 .
$$

For convenience, the following dimensionless variables are introduced:

$$
\widehat{W}=\frac{W}{d} ; \quad \widehat{x}=\frac{x}{l} ; \quad \widehat{t}=\frac{t}{\tilde{t}},
$$

where

$$
\tilde{t}=\sqrt{\frac{\rho b k l^{4}}{E I}} .
$$

Upon substitution of the dimensionless quantities given in (10) into (7) and dropping the hats, one would get

$$
\begin{aligned}
W^{\prime \prime \prime \prime}+\ddot{W}+C_{\mathrm{non}} \dot{W}= & {\left[\alpha_{1} \int_{0}^{1} W^{\prime 2} d x+N\right] W^{\prime \prime} } \\
& +\frac{\beta(t)}{(1-W)^{2}}+\frac{\lambda_{4}}{(1-W)^{4}},
\end{aligned}
$$

where

$$
\beta(t)=\frac{\beta_{0}}{t_{\text {non }}} t U\left(t_{\text {non }}-t\right)+\beta_{0} U\left(t-t_{\text {non }}\right),
$$

where for rectangular cross-section one can obtain

$$
\begin{gathered}
C_{\text {non }}=\frac{12 f l^{4}}{E b k^{3} \widetilde{t}} ; \quad \alpha_{1}=6\left(\frac{d}{k}\right)^{2} ; \quad N=\frac{12 F_{r} l^{2}}{E b k^{3}} ; \\
\beta_{0}=\frac{6 \varepsilon v_{\mathrm{DC}}^{2} l^{4}}{E k^{3} d^{3}} ; \quad \lambda_{4}=\frac{12 l^{4} \pi^{2} \hbar c}{240 E k^{3} d^{5}} ; \\
\alpha_{3}=\frac{12 \rho a_{0} l^{4}}{E d k^{2}} ; \quad t_{\text {non }}=\frac{t_{0}}{\widetilde{t}} .
\end{gathered}
$$

\section{Solution Procedure}

Herein the Galerkin based reduced order modelling is used in order to solve the nonlinear partial differential equation (12) [13]. To this aim, (12) is discretized into a finite degree of freedom system consisting of ordinary differential equations in time. The undamped modeshape of straight clampedclamped nano/microbeam is used as a basis function in Galerkin procedure. To this end, the deflection is expressed as

$$
W(x, t)=\sum_{i=1}^{M} \varphi_{i}(x) u_{i}(t)
$$

where $u_{i}(t)$ is the $i$ th generalized coordinate and $\varphi_{i}(x)$ is the $i$ th linear undamped modeshape of the straight clampedclamped nano/microbeam, normalized such that $\int_{0}^{1} \varphi_{i} \varphi_{j}=$ $\delta_{i j}$ and expressed as [14]

$$
\begin{aligned}
\varphi_{i}(x)= & \cosh \left(\sqrt{\omega_{i}} x\right)-\cos \left(\sqrt{\omega_{i}} x\right) \\
& -\sigma_{n}\left[\sinh \left(\sqrt{\omega_{i}} x\right)-\sin \left(\sqrt{\omega_{i}} x\right)\right],
\end{aligned}
$$

where $\sigma_{n}$ is defined as

$$
\sigma_{n}=\frac{\sinh \left(\sqrt{\omega_{i}}\right)+\sin \left(\sqrt{\omega_{i}}\right)}{\cosh \left(\sqrt{\omega_{i}}\right)-\cos \left(\sqrt{\omega_{i}}\right)}
$$

and $\omega_{i}$ is the $i$ th nondimensional natural frequency of the nano/microbeam and governed by

$$
\cos \left(\sqrt{\omega_{i}}\right) \cosh \left(\sqrt{\omega_{i}}\right)=1
$$

Next we multiply (12) by $\varphi_{n}(x) \cdot(1-W)^{4}$, substitute (15) into the resulting equation, integrate the outcome from $x=0$ to 1 , use integration by parts, and obtain

$$
\begin{aligned}
\ddot{u}_{n} & +C_{\text {non }} \dot{u}_{n}+\left[\omega_{n}^{2}+2 \beta\right] u_{n}-\left[\beta+\lambda_{4}\right] \int_{0}^{1} \varphi_{n} d x \\
& -4 \sum_{i, j=1}^{M} \omega_{i}^{2} u_{i} u_{j} \int_{0}^{1} \varphi_{i} \varphi_{j} \varphi_{n} d x+6 \sum_{i, j, k=1}^{M} \omega_{i}^{2} u_{i} u_{j} u_{k} \\
& \times \int_{0}^{1} \varphi_{i} \varphi_{j} \varphi_{k} \varphi_{n} d x-4 \sum_{i, j, k, m=1}^{M} \omega_{i}^{2} u_{i} u_{j} u_{k} u_{m} \\
& \times \int_{0}^{1} \varphi_{i} \varphi_{j} \varphi_{k} \varphi_{m} \varphi_{n} d x+\sum_{i, j, k, m, p=1}^{M} \omega_{i}^{2} u_{i} u_{j} u_{k} u_{m} u_{p} \\
& \times \int_{0}^{1} \varphi_{i} \varphi_{j} \varphi_{k} \varphi_{m} \varphi_{p} \varphi_{n} d x-4 \sum_{i, j=1}^{M} \ddot{u}_{i} u_{j} \\
& \times \int_{0}^{1} \varphi_{i} \varphi_{j} \varphi_{n} d x+6 \sum_{i, j, k=1}^{M} \ddot{u}_{i} u_{j} u_{k} \int_{0}^{1} \varphi_{i} \varphi_{j} \varphi_{k} \varphi_{n} d x \\
& -4 \sum_{i, j, k, m=1}^{M} \ddot{u}_{i} u_{j} u_{k} u_{m} \int_{0}^{1} \varphi_{i} \varphi_{j} \varphi_{k} \varphi_{m} \varphi_{n} d x \\
& +\sum_{i, j, k, m, p=1}^{M} \ddot{u}_{i} u_{j} u_{k} u_{m} u_{p} \int_{0}^{1} \varphi_{i} \varphi_{j} \varphi_{k} \varphi_{m} \varphi_{p} \varphi_{n} d x
\end{aligned}
$$




$$
\begin{aligned}
& -4 C_{\text {non }} \sum_{i, j=1}^{M} \dot{u}_{i} u_{j} \int_{0}^{1} \varphi_{i} \varphi_{j} \varphi_{n} d x+6 C_{\text {non }} \sum_{i, j, k=1}^{M} \dot{u}_{i} u_{j} u_{k} \\
& \times \int_{0}^{1} \varphi_{i} \varphi_{j} \varphi_{k} \varphi_{n} d x-4 C_{\text {non }} \sum_{i, j, k, m=1}^{M} \dot{u}_{i} u_{j} u_{k} u_{m} \\
& \times \int_{0}^{1} \varphi_{i} \varphi_{j} \varphi_{k} \varphi_{m} \varphi_{n} d x+C_{\text {non }} \sum_{i, j, k, m, p=1}^{M} \dot{u}_{i} u_{j} u_{k} u_{m} u_{p} \\
& \times \int_{0}^{1} \varphi_{i} \varphi_{j} \varphi_{k} \varphi_{m} \varphi_{p} \varphi_{n} d x+\alpha_{1} \sum_{i, j, k=1}^{M} u_{i} u_{j} u_{k} \\
& \times \Gamma\left(\varphi_{i}, \varphi_{j}\right) \Gamma\left(\varphi_{k}, \varphi_{n}\right)+4 \alpha_{1} \sum_{i, j, k, m=1}^{M} u_{i} u_{j} u_{k} u_{m} \Gamma\left(\varphi_{i}, \varphi_{j}\right) \\
& \times \int_{0}^{1} \varphi_{k}^{\prime \prime} \varphi_{m} \varphi_{n} d x-6 \alpha_{1} \sum_{i, j, k, m, p=1}^{M} u_{i} u_{j} u_{k} u_{m} u_{p} \\
& \times \Gamma\left(\varphi_{i}, \varphi_{j}\right) \int_{0}^{1} \varphi_{k}^{\prime \prime} \varphi_{m} \varphi_{p} \varphi_{n} d x+4 \alpha_{1} \\
& \times \sum_{i, j, k, m, p, q=1}^{M} u_{i} u_{j} u_{k} u_{m} u_{p} u_{q} \Gamma\left(\varphi_{i}, \varphi_{j}\right) \int_{0}^{1} \varphi_{k}^{\prime \prime} \varphi_{m} \varphi_{p} \varphi_{q} \varphi_{n} d x \\
& -\alpha_{1} \sum_{i, j, k, m, p, q, l=1}^{M} u_{i} u_{j} u_{k} u_{m} u_{p} u_{q} u_{l} \Gamma\left(\varphi_{i}, \varphi_{j}\right) \\
& \times \int_{0}^{1} \varphi_{k}^{\prime \prime} \varphi_{m} \varphi_{p} \varphi_{q} \varphi_{l} \varphi_{n} d x+N \sum_{i=1}^{M} u_{i} \Gamma\left(\varphi_{i}, \varphi_{n}\right) \\
& +4 N \sum_{i, j=1}^{M} u_{i} u_{j} \int_{0}^{1} \varphi_{i}^{\prime \prime} \varphi_{j} \varphi_{n} d x-6 N \sum_{i, j, k=1}^{M} u_{i} u_{j} u_{k} \\
& \times \int_{0}^{1} \varphi_{i}^{\prime \prime} \varphi_{j} \varphi_{k} \varphi_{n} d x+4 N \sum_{i, j, k, m=1}^{M} u_{i} u_{j} u_{k} u_{m} \\
& \times \int_{0}^{1} \varphi_{i}^{\prime \prime} \varphi_{j} \varphi_{k} \varphi_{m} \varphi_{n} d x-N \sum_{i, j, k, m, p=1}^{M} u_{i} u_{j} u_{k} u_{m} u_{p} \\
& \times \int_{0}^{1} \varphi_{i}^{\prime \prime} \varphi_{j} \varphi_{k} \varphi_{m} \varphi_{p} \varphi_{n} d x-\beta \sum_{i, j=1}^{M} u_{i} u_{j} \int_{0}^{1} \varphi_{i} \varphi_{j} \varphi_{n} d x=0
\end{aligned}
$$

where the function $\Gamma\left(\varphi_{i}, \varphi_{j}\right)$ is given by

$$
\Gamma\left(\varphi_{i}, \varphi_{j}\right)=\int_{0}^{1} \varphi_{i}^{\prime} \varphi_{j}^{\prime} d x
$$

Equation (19) represents an implicit system of $M$ nonlinear second order ordinary differential equations. Using an implicit scheme such as Adams-Moulton implicit methods [15] or transforming them into an explicit system in $\ddot{u}_{n}$ by multiplying (19) with the inverse of the coefficients of $\ddot{u}_{n}$

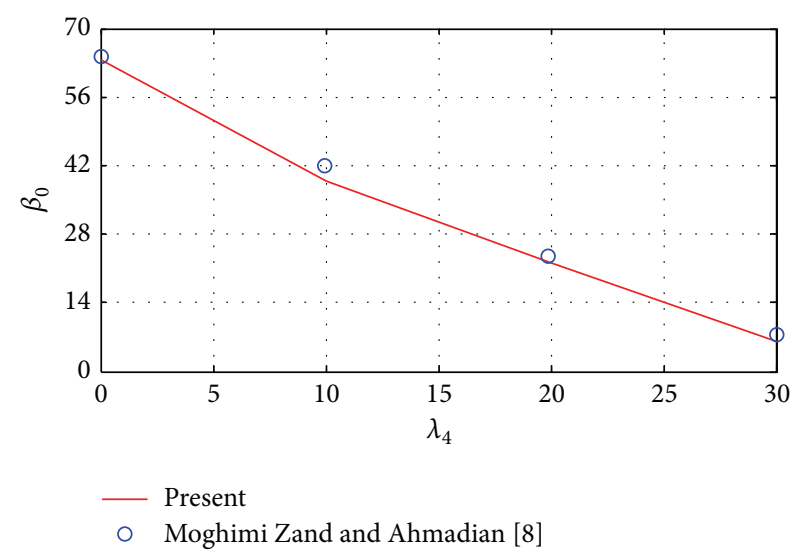

FIgUre 3: Nondimensional Casimir parameter versus the nondimensional parameter of maximum value for the case in which $\alpha_{1}=$ $6, N=0, C_{\text {non }}=0$, and $t_{0}=10^{-7} \mathrm{sec}$.

and using fourth order Runge-Kutta method may lead to the solution of (19). The latter approach was used in the present calculations.

\section{Results and Discussion}

Assuming that the only dominant mode in the response of the nano/microbeam is its first mode (i.e., $M=1$ ), so using the single mode approximation may lead to accurate results. In order to validate the model, the results are compared with those presented in the literature for DC dynamic pullin case in which the Casimir effect has been taken into account [8]. For this aim we set $\alpha_{1}=6, N=0, C_{\text {non }}=$ 0 , and $t_{0}=10^{-7} \mathrm{sec}$, then plot the nondimensional max voltage parameter $\beta_{0}$ versus nondimensional Casimir force parameter $\lambda_{4}$, and compare the outcome with the results of Moghimi Zand and Ahmadian [8] (see Figure 3).

Consider a polysilicon microbeam with length $l=$ $900 \mu \mathrm{m}$, thickness $k=1.5 \mu \mathrm{m}$, width $b=100 \mu \mathrm{m}$ and gap width $d=2 \mu \mathrm{m}$. The material properties of this microbeam are $v=0.28, E=169 \mathrm{GPa}$ which is replaced by $E /(1-$ $v^{2}$ ) because $b>5 k$, and $\rho=2332 \mathrm{Kg} / \mathrm{m}^{3}$. The DC dynamic pull-in step voltage for this case without considering damping coefficient and Casimir effect is $3.11 \mathrm{~V}$. The response of this microbeam under ramp-input voltage with and without considering the effect of Casimir force before and after bechancing pull-in instability is plotted in Figure 4 . From this figure, one can observe the major effect of Casimir force on nano/microstructures.

Figure 4 illustrated that neglecting the effect of Casimir force on nano/microstructures may lead to very inaccurate results, so it is very important to consider this effect when the nondimensional Casimir parameter $\left(\lambda_{4}\right)$ takes noticeable values.

\section{Conclusion}

In the present paper, reduced order modelling based on the Galerkin procedure was utilized to study the effect 


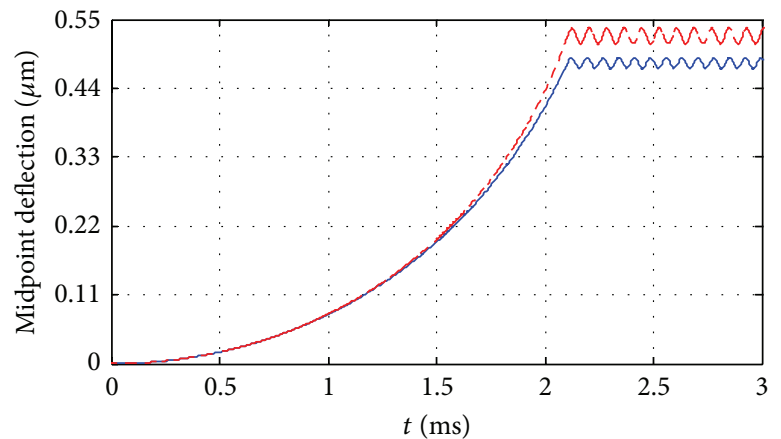

— Without the effect of Casimir force

- - - With the effect of Casimir force

(a)

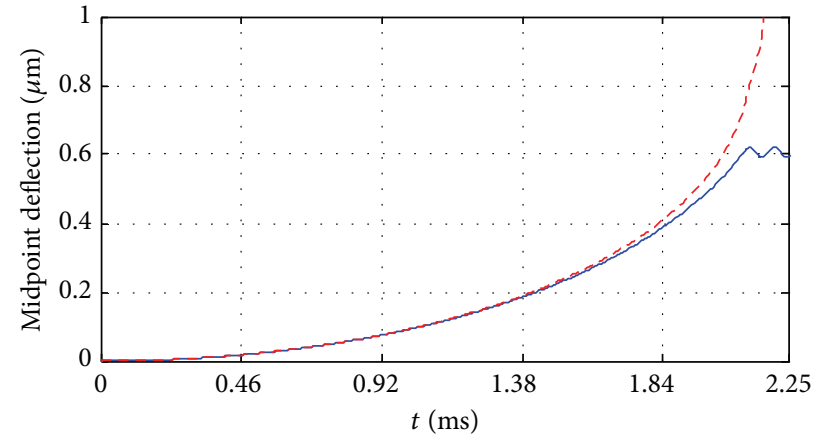

Without the effect of Casimir force, $v_{0}=3.2 \mathrm{~V}, t_{\text {non }}=10$ - - - With the effect of Casimir force, $v_{0}=3.2 \mathrm{~V}, t_{\text {non }}=10$

(b)

Figure 4: The response of polysilicon microbeam under combined effect of ramp-input voltage and the Casimir force force. (a) $v_{0}=3 \mathrm{~V}$, $t_{\text {non }}=10$ and (b) $v_{0}=3.2 \mathrm{~V}, t_{\text {non }}=10$.

of Casimir attraction on the response of a fully clamped nano/microbeam under ramp-input voltage. The model accounted for geometric nonlinearity of von Kármán midplane stretching, applied axial loading, equivalent viscose damping, and inherent nonlinearity of distributed electrostatic and Casimir forces. It was found that considering the Casimir force may lead to early instability in nano/microelectromechanical devices through dynamic pull-in.

\section{Conflict of Interests}

The authors declare that there is no conflict of interests regarding the publication of this paper.

\section{References}

[1] H. C. Nathanson, W. E. Newell, R. A. Wickstrom, and J. R. Davis, "The resonant gate transistor," IEEE Transactions on Electron Devices, vol. 14, no. 3, pp. 117-133, 1967.

[2] G. I. Taylor, "The coalescence of closely spaced drops when they are at different electric potentials," Proceedings of the Royal Society A, vol. 306, pp. 423-434, 1968.

[3] H. B. G. Casimir, "On the attraction between two perfectly conducting plates," in Proceedings of the Koninklijke Nederlandse Akademie van Wetenschappen, vol. 51, pp. 793-795, 1948.

[4] F. M. Serry, D. Walliser, and G. J. Maclay, "Anharmonic casimir oscillator (ACO) - the casimir effect in a model microelectromechanical system," Journal of Microelectromechanical Systems, vol. 4, no. 4, pp. 193-205, 1995.

[5] W.-H. Lin and Y.-P. Zhao, "Nonlinear behavior for nanoscale electrostatic actuators with Casimir force," Chaos, Solitons and Fractals, vol. 23, no. 5, pp. 1777-1785, 2005.

[6] A. Ramezani, A. Alasty, and J. Akbari, "Closed-form solutions of the pull-in instability in nano-cantilevers under electrostatic and intermolecular surface forces," International Journal of Solids and Structures, vol. 44, no. 14-15, pp. 4925-4941, 2007.

[7] A. Koochi, A. S. Kazemi, Y. Tadi Beni, A. Yekrangi, and M. Abadyan, "Theoretical study of the effect of Casimir attraction on the pull-in behavior of beam-type NEMS using modified Adomian method," Physica E, vol. 43, no. 2, pp. 625-632, 2010.
[8] M. Moghimi Zand and M. T. Ahmadian, "Dynamic pull-in instability of electrostatically actuated beams incorporating Casimir and van der Waals forces," Journal of Mechanical Engineering Science, vol. 224, no. 9, pp. 2037-2047, 2010.

[9] S. K. Lamoreaux, "The Casimir force: background, experiments, and applications," Reports on Progress in Physics, vol. 68, no. 1, pp. 201-236, 2005.

[10] J. Qian, C. Liu, D. Zhang, and Y. Zhao, "Residual stresses in micro-electro-mechanical systems," Journal of Mechanical Strength, vol. 23, no. 4, pp. 393-401, 2001.

[11] L. D. Landau and E. M. Lifshitz, Theory of Elasticity, Pergamon Press, New York, NY, USA, 1986.

[12] A. H. Nayfeh and M. I. Younis, "A new approach to the modeling and simulation of flexible microstructures under the effect of squeeze-film damping," Journal of Micromechanics and Microengineering, vol. 14, no. 2, pp. 170-181, 2004.

[13] M. I. Younis, E. M. Abdel-Rahman, and A. Nayfeh, "A reducedorder model for electrically actuated microbeam-based MEMS," Journal of Microelectromechanical Systems, vol. 12, no. 5, pp. 672-680, 2003.

[14] B. Balachandran and E. Magrab, Vibrations, Cengage Learning, Toronto, Canada, 2nd edition, 2009.

[15] J. D. Faires and R. L. Burden, Numerical Methods, Faires and Burden, Brooks/Cole, 3rd edition, 2002. 

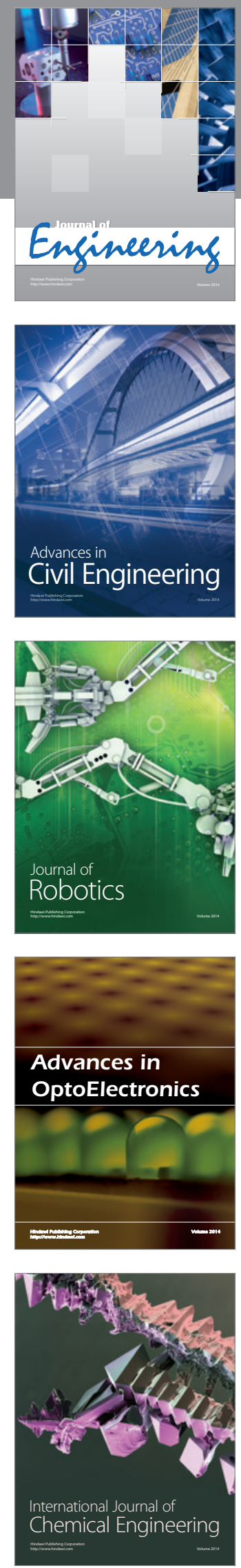

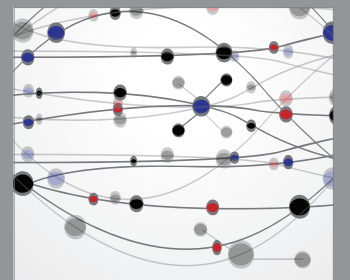

The Scientific World Journal
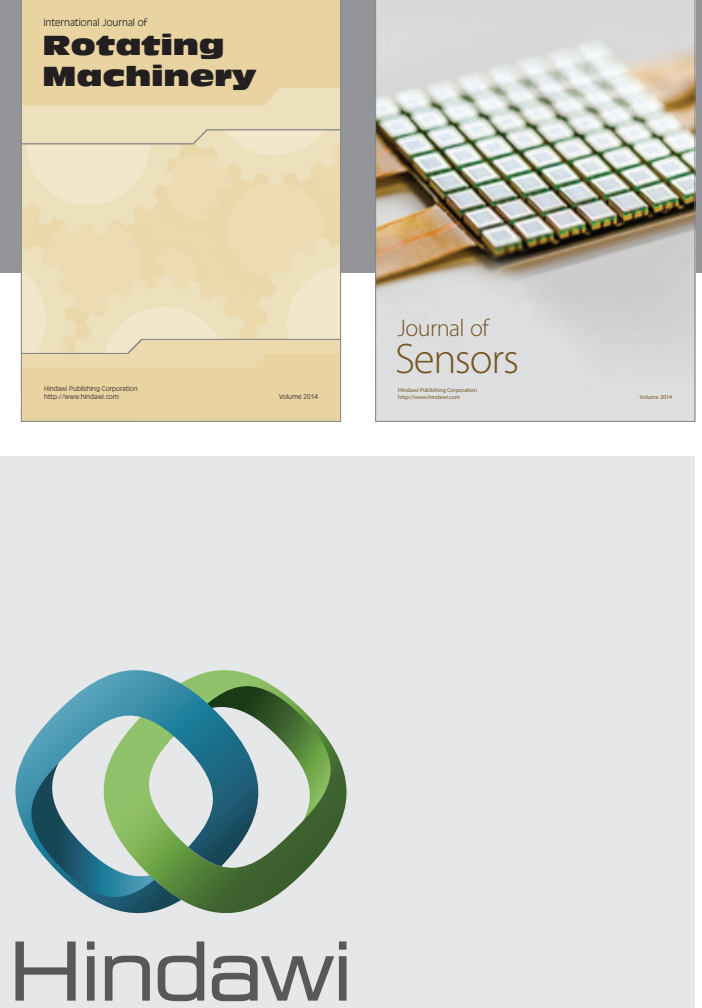

Submit your manuscripts at http://www.hindawi.com
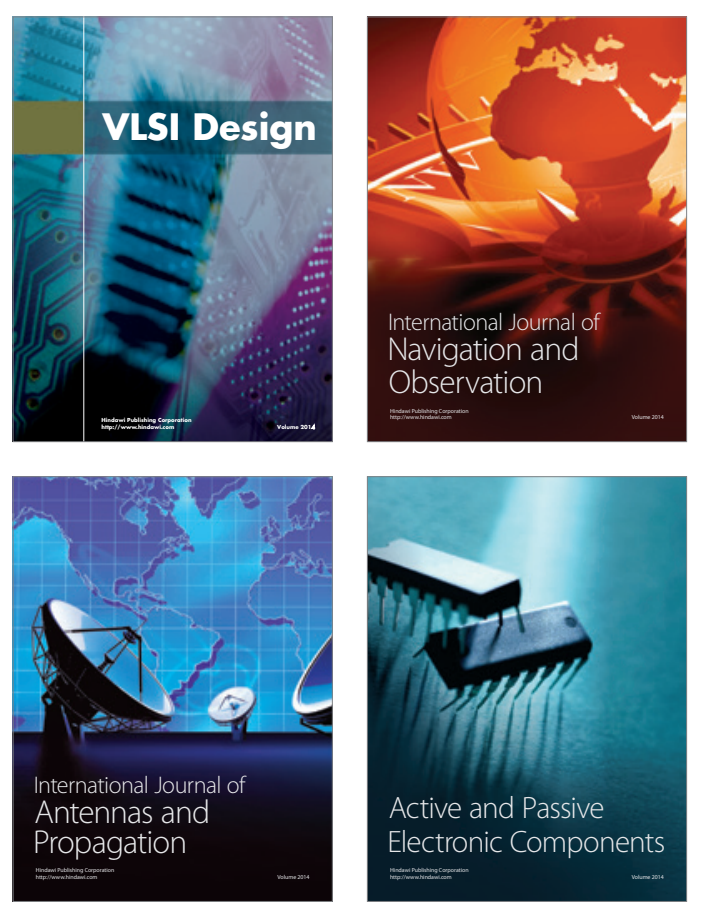
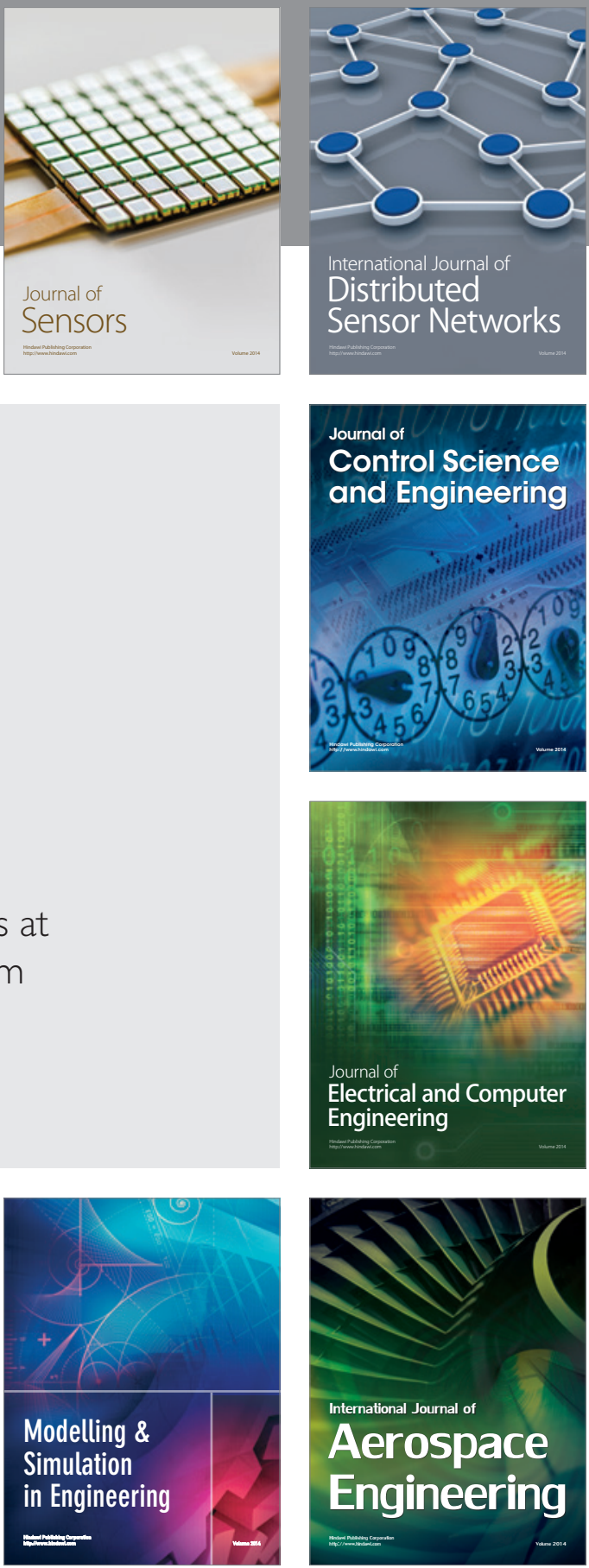

Journal of

Control Science

and Engineering
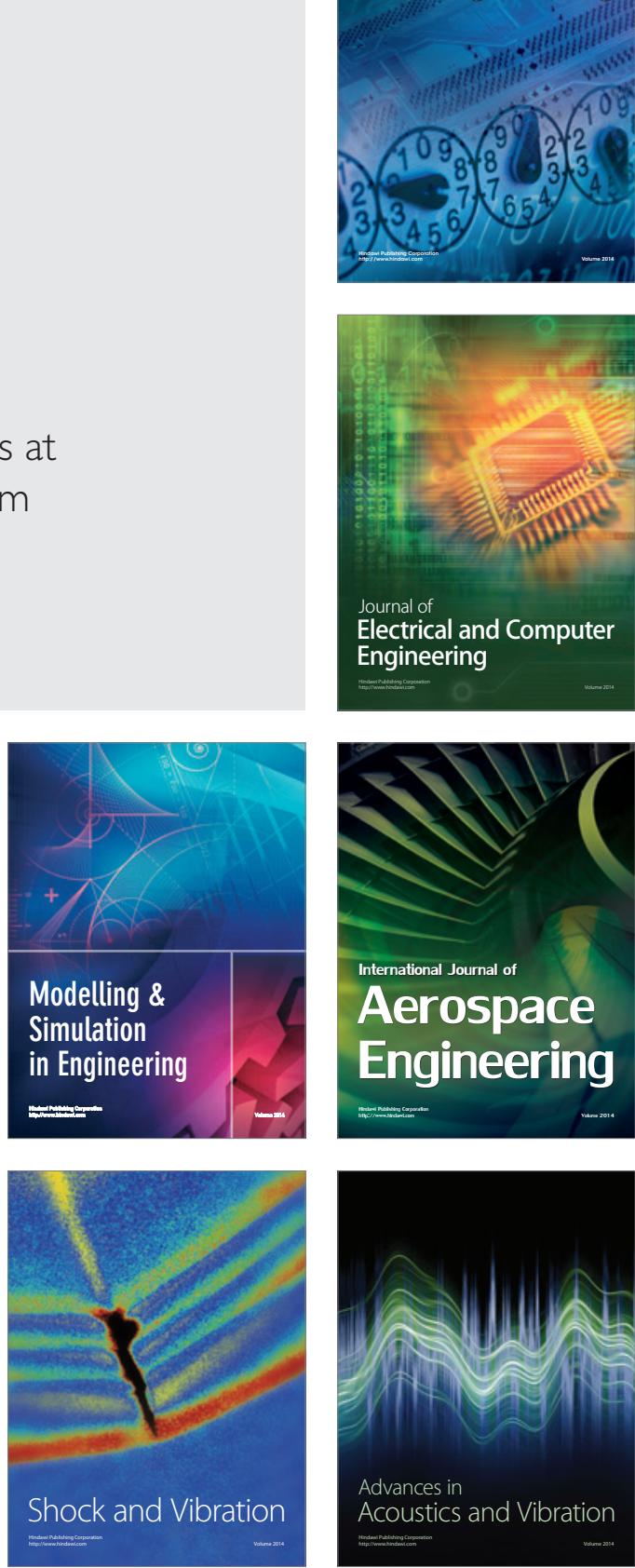